\title{
LINEAR QUADRATIC OPTIMAL CONTROL: FROM DETERMINISTIC TO STOCHASTIC CASES
}

Shuping Chen*

Department of Applied Mathematics Zhejiang University, Hangzhou 310027, China.

Xinhui Yu

Institute of Mathematics

Fudan University, Shanghai 200433, China

\section{DETERMINISTIC LQ PROBLEM: A BRIEF HISTORICAL OVERVIEW}

Given the following linear system

$$
\dot{x}(t)=A x(t)+B u(t), \quad x(0)=x_{0} .
$$

and the cost functional

$$
J\left(x_{0}, u(\cdot)\right)=\langle G x(T), x(T)\rangle+\int_{0}^{T}[\langle Q x(t), x(t)\rangle+\langle R u(t), u(t)\rangle] d t
$$

where $x_{0} \in \mathbb{R}^{n}, x(t) \in \mathbb{R}^{n}$ and $u(t) \in \mathbb{R}^{m}$ for all $t \geq 0, A, B, G, Q, R$ are matrices of correspondent dimension, and $G, Q, R$ are symmetric. Our problem is:

Problem (DLQ). Minimize (1.2), subject to (1.1).

In the optimal control theory, the above problem is referred to as deterministic linear quadratic optimal control problem (DLQ problem, for short). Following are two definitions associated with it. Problem (DLQ) is said to be finite if

$$
\forall x_{0} \in \mathbb{R}^{n}, \quad V\left(x_{0}\right) \triangleq \inf _{u(\cdot) \in L^{2}\left(0, T ; \mathbf{R}^{m}\right)} J\left(x_{0}, u(\cdot)\right)>-\infty .
$$

*This work is supported in part by the NSFC, under grant 79790130.

The original version of this chapter was revised: The copyright line was incorrect. This has been corrected. The Erratum to this chapter is available at DOI: 10.1007/978-0-387-35359-3_40 
Problem (DLQ) is said to be (uniquely) solvable if

$$
\forall x_{0} \in \mathbb{R}^{n}, \exists \bar{u}(\cdot) \in L^{2}\left(0, T ; \mathbb{R}^{m}\right) \text {, s.t. } V\left(x_{0}\right)=J\left(x_{0}, \bar{u}(\cdot)\right) .
$$

LQ problem has roots going back to the very beginning of optimal control theory, the fundamental work was due to R. E. Kalman [5] in 1960. Since then, a lot of works have been done on DLQ problem, among them, we mention A. M. Letov [7], J. C. Willems [11], B. P. Molinari [10] and V. A. Yakubovic [13] etc. for finite dimensional cases, and I. Lasiecka and R. Triggiani [6], X. Li and J. Yong [8], S. Chen [2] and J. L. Lions [9] for infinite dimensional cases.

By now, it is very well known that

- If Problem (DLQ) is finite, by which, we mean that the infimum of the cost functional is finite, then $R \geq 0$.

- If $R \geq 0$, then Problem (DLQ) is (uniquely) solvable if and only if

$$
\begin{cases}\dot{x}(t)=A x(t)+B u(t), & x(0)=x_{0} \\ \dot{p}(t)=-Q x(t)-A^{T} p(t), & p(T)=G x(T), \\ R u(t)+B^{T} p(t)=0, & \end{cases}
$$

admits a (unique) solution $(x, u, p)$.

- If $R>0$ and Riccati equation

$$
\dot{P}=-\left(P A+A^{T} P+Q\right)+P B^{T} R^{-1} B P, \quad P(T)=G .
$$

admits a solution $P(\cdot)$, then Problem (DLQ) is uniquely solvable with the optimal control

$$
\bar{u}(t)=-R^{-1} B^{T} P \bar{x}(t) .
$$

- If $Q \geq 0, G \geq 0, R>0$, then Problem (DLQ) is uniquely solvable.

\section{STOCHASTIC LQ PROBLEM: A NEW PHENOMENON}

Let $\left(\Omega, \mathcal{F}, \mathcal{P},\left\{\mathcal{F}_{t}\right\}_{t \geq 0}\right)$ be a complete filtered probability space on which defined a one dimensional standard Brownian motion $w(\cdot)$ such that $\left\{\mathcal{F}_{t}\right\}_{t \geq 0}$ is the natural filteration generated by $w(\cdot)$, augmented by all the $\mathcal{P}$-null sets in $\mathcal{F}$. Consider the following linear controlled stochastic diffenrential equation:

$$
d x(t)=[A x(t)+B u(t)] d t+[C x(t)+D u(t)] d w(t), \quad x(\tau)=\xi
$$

and the cost functional

$$
J(\tau, \xi ; u(\cdot))=E\left\{\langle G x(T), x(T)\rangle+\int_{\tau}^{T}\{\langle Q x(t), x(t)\rangle+\langle R u(t), u(t)\rangle\} d t\right\},
$$

where $\tau \in \mathcal{T}[0, T]$, the set of all $\left\{\mathcal{F}_{t}\right\}_{t \geq 0}$-stopping times taking values in $[0, T], \xi \in \chi_{\tau} \triangleq L_{\mathcal{F}_{\tau}}^{2}\left(\Omega, \mathbb{R}^{n}\right)$, the set of all $\mathbb{R}^{n}$-valued $\mathcal{F}_{\tau}$-measurable squareintegrable random variables; $A, B, C, D$ are matrix-valued $\left\{\mathcal{F}_{t}\right\}_{t \geq 0 \text {-adapted }}$ 
bounded processes, $Q, R$ are symmetric matrix-valued $\left\{\mathcal{F}_{t}\right\}_{t \geq 0}$-adapted bounded processes, $G$ is symmetric matrix-valued $\mathcal{F}_{T}$-measurable bounded random variable, $u(\cdot)$ is a control process and $x(\cdot)$ is the corresponding state process. Our problem is:

Problem (SLQ). Minimize (2.2) subject to (2.1).

Here, our control process $u(\cdot)$ is taken from $\mathcal{U}[\tau, T]=L_{\mathcal{F}}^{2}\left(\tau, T ; \mathbb{R}^{m}\right)$, the set of all $\mathbb{R}^{m}$-valued $\left\{\mathcal{F}_{t}\right\}_{t \geq 0}$-adapted square-integrable processes defined on the random interval $[\tau, T]$ with $\tau \in \mathcal{T}[0, T]$. Let $\Delta[0, T]=\cup_{r \in \mathcal{T}[0, T]}\left[\{r\} \times \chi_{r}\right]$, $V(\tau, \xi)=\inf _{u(\cdot) \in \mathcal{U}[\tau, T]} J(\tau, \xi, u(\cdot))$. Then one has

$$
V: \Delta[0, t] \rightarrow \mathbb{R} .
$$

Problem (SLQ) has recently been extensively studied in [3, 4], which is referred to as the stochastic linear quadratic optimal control problem (SLQ problem, for short) with random coefficient. Problem (SLQ) is said to be finite at $(\tau, \xi) \in \Delta[0, T]$, if

$$
V(\tau, \xi)>-\infty
$$

Problem (SLQ) is said to be solvable at $(\tau, \xi) \in \Delta[0, T]$, if there exists a $\bar{u}(\cdot)$, such that

$$
J(\tau, \xi ; \bar{u}(\cdot))=V(\tau, \xi) .
$$

Comparing with its deterministic counterpart, Problem (SLQ) have some new interesting features. Firstly, in the deterministic case, $R \geq 0$ is a necessary condition for the finiteness; but in the stochastic case, $R \geq 0$ is no longer necessary for the finiteness. Example 2.1 shows that SLQ problem with $R<0$ may uniquely solvable. Secondly, $R>0$ can assure the unique solvability of Problem (DLQ), but can't even assure the finiteness of Problem (SLQ) sometimes. Example 2.2 shows this fact.

Example 2.1 Consider:

$$
\dot{x}(t)=u(t), \quad x(0)=x_{0} \in \mathbb{R},
$$

with the cost functional

$$
J(u(\cdot))=x(T)^{2}-\int_{0}^{T} u(t)^{2} d t .
$$

Thus, $R=-1<0$.

$$
\inf _{u(\cdot)} J(u(\cdot))=-\infty .
$$

Consider the stochastic version:

$$
d x(t)=u(t) d t+\delta u(t) d w(t), \quad x(0)=x_{0},
$$

with the cost functional

$$
J(u(\cdot))=E\left\{x(T)^{2}-\int_{0}^{T} u(t)^{2} d t\right\} .
$$


It can be proved that, If $|\delta|>0$ and

$$
\delta^{2}(2 \ln |\delta|-1)>T-1
$$

then Problem (SLQ) is solvable on $[0, T]$.

Example 2.2 Consider: $(0<T<1)$

$$
\dot{x}(t)=u(t), \quad x(0)=x_{0} \in \mathbb{R} .
$$

with the cost functional

$$
J(u(\cdot))=-x(T)^{2}+\int_{0}^{T} u(t)^{2} d t .
$$

Thus, $R=1>0$. We can show that Problem (DLQ) is uniquely solvable with optimal control:

$$
\bar{u}(t)=\frac{x(t)}{t+1-T} .
$$

Now, consider the stochastic version:

$$
d x(t)=u(t) d t+\delta u(t) d w(t), \quad x(0)=x_{0} .
$$

with the cost functional

$$
J(u(\cdot))=E\left\{-x(T)^{2}+\int_{0}^{T} u(t)^{2} d t\right\} .
$$

It can be proved: If $|\delta|>1$, then Problem (SLQ) is not finite.

Then one will ask: what's the necessary condition for the finiteness of Problem $(S L Q)$ ? The following theorem answer this.

Theorem 2.1 ([4]) If Problem (SLQ) is finite at some $(\tau, \xi) \in \Delta[0, T]$, then

$$
R(T)+D^{T}(T) G D(T) \geq 0, \quad \text { a.s. } \omega \in \Omega .
$$

From Theorem 2.1 and the arguments above, we can easy see that $D$ plays a very interesting role in the finiteness and solvability of problem (SLQ). When $A, B, C, D, G, Q, R$ are matrices, (2.3) becomes

$$
R+D^{T} G D \geq 0 \text {. }
$$

In this case, if $D=0$, i.e., control does not appear in the diffusion, (2.4) becomes $R \geq 0$, the same as the DLQ problem. In Example 2.2,

$$
R+D^{T} G D=1-\delta^{2} .
$$

Thus, $\delta>1$ violates the necessary condition. 


\section{STOCHASTIC MAXIMUM PRINCIPLE AND STOCHASTIC RICCATI EQUATION}

The following forward-backward stochastic differential equation (FBSDE, for short)

$$
\left\{\begin{array}{l}
d x(t)=[A x(t)+B u(t)] d t+[C x(t)+D u(t)] d w(t) \\
d p(t)=-\left[A^{T} p(t)+C^{T} q(t)+Q x(t)\right] d t+q(t) d w(t) \\
x(\tau)=\xi, \quad p(T)=G x(T)
\end{array}\right.
$$

will play a very important role in the stochastic maximun principle:

Theorem 3.1 ([4]) Problem (SLQ) is solvable at $(\tau, \xi) \in \Delta[0, T]$, with an optimal pair $(\bar{x}(\cdot), \bar{u}(\cdot))$ if and only if the following forward-backward stochastic differential equation (FBSDE)

$$
\left\{\begin{array}{l}
d \bar{x}(t)=[A \bar{x}(t)+B \bar{u}(t)] d t+[C \bar{x}(t)+D \bar{u}(t)] d w(t), \\
d \bar{p}(t)=-\left[A^{T} \bar{p}(t)+C^{T} \bar{q}(t)+Q \bar{x}(t)\right] d t+\bar{q}(t) d w(t), \\
\bar{x}(\tau)=\xi, \quad \bar{p}(T)=G \bar{x}(T)
\end{array}\right.
$$

admits an adapted solution $(\bar{x}(\cdot), \bar{p}(\cdot), \bar{q}(\cdot))$ such that

$$
I_{\Omega(\tau, \xi)}\left[R \bar{u}(t)+B^{T} \bar{p}(t)+D^{T} \bar{q}(t)\right]=0, \quad \text { in } L_{\mathcal{F}}^{2}\left(\tau, T ; \mathbb{R}^{m}\right),
$$

and for any $u(\cdot) \in \mathcal{U}[\tau, T]$, the unique adapted solution $(x(\cdot), p(\cdot), q(\cdot))$ of (3.1) with $\xi=0$ satisfies

$$
E \int_{\tau}^{T}\left\langle R u(t)+B^{T} p(t)+D^{T} q(t), u(t)\right\rangle d t \geq 0
$$

Another important thing associated with the solvability of Problem (SLQ) is the following stochastic backward Riccati equation for symmetric matrix-valued process $(P(\cdot), \Lambda(\cdot))$ in a random time interval:

$$
\left\{\begin{array}{c}
d P=-\left\{P A+A^{T} P+C^{T} P C+\Lambda C+C^{T} \Lambda+Q\right. \\
-\left(P B^{T}+C^{T} P D+\Lambda D\right)\left(R+D^{T} P D\right)^{-1} \\
\left.\cdot\left(B^{T} P+D^{T} P C+D^{T} \Lambda\right)\right\} d t+\Lambda d w(t), \quad t \in[\tau, T] \\
P(T)=G . \\
\operatorname{det}\left[R(t)+D(t)^{T} P(t) D(t)\right] \neq 0, \quad t \in[\tau, T], \text { a.s. } \omega \in \Omega
\end{array}\right.
$$

where $\tau \in \mathcal{T}[0 . T]$.

Theorem $3.2([3,4])$ If $(3.5)$ admits an adapted solution $(P, \Lambda)$, then Problem $(S L Q)$ is uniquely solvable at $(\tau, \xi)$, with the optimal control

$$
u(t)=-\left(R+D^{T} P D\right)^{-1}\left(B^{T} P+D^{T} P C+D^{T} \Lambda\right) x(t) .
$$




\section{SOLVABILITY OF RICCATI EQUATION WITH DETERMINISTIC COEFFICIENT}

Now, we consider Problem (SLQ) with deterministic coefficient, i.e., $\tau \in[0, T]$, $\xi \in \mathbb{R}^{n}, A, C, Q, G \in \mathbb{R}^{n \times n}, B, D \in \mathbb{R}^{n \times m}, R \in \mathbb{R}^{m \times m}$. In this case, the stochastic maximum principle is as following

Theorem 4.1 ([4]) Let Problem (SLQ) be uniquely solvable. Then (3.1) admits a solution satisfies

$$
R u(t)+B^{T} p(t)+D^{T} q(t)=0
$$

Conversely, if (3.1)-(3.2) admits a (unique) solution $(\bar{x}, \bar{u}, \bar{p}, \bar{q})$, and any adapted solution $(x, u, p, q)$ of (3.1) with $\xi=0$ satisfies

$$
E \int_{0}^{T}\left\langle R u(t)+B^{T} p(t)+D^{T} q(t), u(t)\right\rangle d t \geq 0
$$

then Problem (SDLQ) is (uniquely) solvable; in addition, if $R^{-1}$ exists, then

$$
\bar{u}(t)=-R^{-1}\left[B^{T} \bar{p}(t)+D^{T} \bar{q}(t)\right],
$$

is the optimal control.

The stochastic backward Riccati equation (3.5) becomes:

$$
\left\{\begin{aligned}
& P=-\left(P A+A^{T} P+C^{T} P C+Q\right) \\
&+\left(P B^{T}+C^{T} P D\right)\left(R+D^{T} P D\right)^{-1}\left(B^{T} P+D^{T} P C\right), \quad t \in[\tau, T] \\
& P(T)=G
\end{aligned}\right.
$$

For Riccati equation (4.4), we have (see [3, 4])

(1) If $R>0, Q \geq 0, G \geq 0$, then (4.4) is globally solvable.

(2) If $C=0$. Then Riccati equation (4.4) is globally solvable if and only if there exists $K^{ \pm}$, such that

$$
K^{+} \geq R+D^{T} \Psi\left(K^{+}\right) \geq R+D^{T} \Psi\left(K^{-}\right) D \geq K^{-},
$$

where $\Psi(K)$ is the solution of

$$
\left\{\begin{array}{l}
\dot{P}=-\left(P A+A^{T} P+C^{T} P C+Q\right) \\
\quad+\left(P B^{T}+C^{T} P D\right) K^{-1}\left(B^{T} P+D^{T} P C\right) \\
P(T)=G
\end{array}\right.
$$

\section{References}

[1] A. Bensoussan, Lecture on Stochastic Control, part 1, Lecture Notes in Math., 972(1983), 1-39.

[2] S. Chen, Riccai Equations Arising in Infinite Dimensional Optimal Control Problems, Control Theory Appl. , 2(1985), 64-72. 
[3] S. Chen, X. Li and X. Y. Zhou, Stochastic Linear Quadratic Regulators with Indefinite Control Weight Costs, SIAM J. Control and Optim., toappear.

[4] S. Chen and J. Yong, Stochastic Linear Quadratic Optimal Control Problems, I,II, preprint.

[5] R. E. Kalman, Contributions to the Theory of Optimal Control, Bol. Soc. Mat. Mex., 5(1960), 102-199.

[6] I. Lasiecka and R.Triggiani, Differential and Algebraic Riccati Equations with Applications to Boundary/Point Control Problems: Continuous Theory and Approximation Theory, Lecture Notes in Control \& Inform. Sci., Vol. 164, Springer-Verlag, Berlin, 1991.

[7] A. M. Letov, Analytical Design of Regulators, Avtomat. i Telemekh. 4(1960), 436-446; 5(1960), 561-571; 6(1960), 661-669. English transl. in Automat. Remote Control, 21(1960).

[8] X. Li and J.Yong, Optimal Control Theory for Infinite Dimensional System, Birkhäuser, Boston, 1995.

[9] J. L. Lions, Optimal Control of Systems Governed by Partial Differential Equations, Springer-Verlag, New York, 1971.

[10] B. P. Molinari, The Time-invariant Linear-Quadratic Optimal Control Problem, Automatica, 13(1977), 347-357.

[11] J. C. Willems, Least Squares Stationary Optimal Control and the Algebraic Riccati Equation, IEEE Trans. Auto. Control, 16(1971), 621-634.

[12] W. M. Wonham, On A Matrix Riccati Equation of Stochastic Control, SIAM J. Control, 6(1968), 312-326.

[13] V. A. Yakubovic, Singular Stationary Nonhomogeneous Linear Quadratic Optimal Control, Amer. Math. Transl., 155(1993), 129-167. 